\title{
Neue Ergebnisse über die Arbeitshyperthermie des Menschen
}

\author{
Jürgen Kitzing und Adolf Bleichert \\ Physiologisches Institut der Universität Hamburg, Hamburg
}

\begin{abstract}
New results on exercise hyperthermia of man. NIELSEN (1938) demonstrated that hypothermia during exercise is independent of room temperature within a range from $5^{\circ}$ to $32^{\circ} \mathrm{C}$. Subsequently, other investigators confirmed this observation. From these results, AsmusSEN \& NIELSEN (1947) concluded that a resetting of the thermoregulatory centre brought about by impulses reaching the brain from the working muscles or from the motor centres takes place. In order to find out whether impulses from motor centres really affect the thermoregulatory centres, we tried, by administration of curare in human beings, to increase the frequency of impulses necessary to bring about a certain amount of mechanical work. However, in 2 series of experiments at room temperatures of $23^{\circ}$ and $34^{\circ} \mathrm{C}$ respectively and at a constant work output of $3 \mathrm{mkp} / \mathrm{sec}$, no significant differences in body temperature (measured in the lower esophagus) before and during curare infusion could be detected. In the resting conditions, curare exerts no influence on body temperature. In normal subjects performing low work $(3 \mathrm{mkp} / \mathrm{sec})$ the body temperature decreases at a room temperature of $23^{\circ} \mathrm{C}$ but increases at a room temperature of $32^{\circ} \mathrm{C}$. In conclusion, the hyperthermia during exercise cannot be due to a resetring of thermoregulatory centres. It works rather like a proportional closed loop control system. The relative independence of the hyperthermia from the environmental temperature suggests a considerable influence of the latter parameter via the thermoreceptors of the skin on the thermoregulation during work.
\end{abstract}

\section{EINLEITUNG}

Seit NielseNs Untersuchungen von 1938 wird die erhöhte Körpertemperatur bei Muskelarbeit als Folge einer regulativen Höherstellung temperaturempfindlicher Zentren aufgefaßt. NIELSEN begründet seine Theorie mit den Tatsachen, daß die erreichte Körpertemperatur der geleisteten mechanischen Arbeit proportional und in weiten Grenzen unabhängig von den klimatischen Umgebungsbedingungen ist. Als mögliche Ursache dieser Verstellung der Zentren nehmen AsMussen und NIELsEN (1947) eine Irradiation efferenter Impulse von der motorischen Hirnrinde oder eine Summation proprioceptiver Impulse aus der arbeitenden Muskulatur an. Eine Summation proprioceptiver Impulse scheint nach den Versuchen von RoBINson (1962) unwahrscheinlich, denn bei heftiger passiver Arbeit wächst die Kerntemperatur nur entsprechend der geringfügigen Stoffwechselsteigerung an. 


\section{ERGEBNISSE UND SCHLUSSFOLGERUNGEN}

Um zu klären, inwieweit eine Irradiation als Ursache des Temperaturanstieges in Frage kommt, haben wir versucht - analog den Uberlegungen von OCrWwaDT et al. (1959) - mit Hilfe eines partiellen neuromuskulären Blocks durch d-Tubocurarinchlorid den Innervationsaufwand bei konstant gehaltener Arbeit zu vergrößern. Im

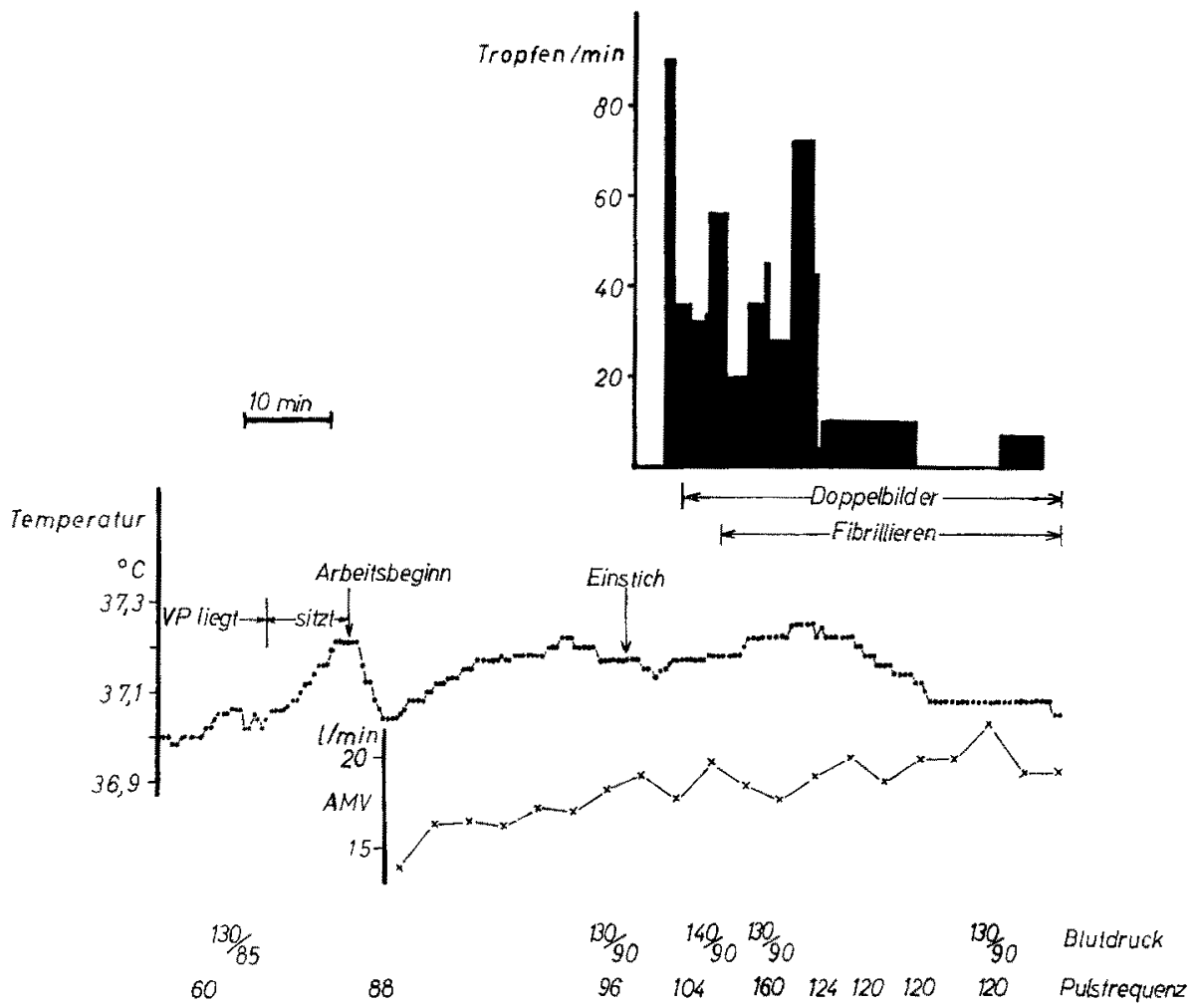

Abb. 1: Beispiel eines Arbeitsversuchs mit Infusion von d-Tubocurarinchlorid. Vp.: V.R. 24 Jahre. Eingestellte Leistung $3 \mathrm{mkp} / \mathrm{sec}$. Raumtemperatur $23^{\circ} \mathrm{C}$, relative Lufffeuchtigkeit $70 \%$. Gesamtdosis: $12 \mathrm{mg}$ d-Tubocurarinchlorid i. v. Blutdruck vor dem Versuch $130 / 80 \mathrm{~mm}$ Hg. Pulsfrequenz vor dem Versuch 60/min. Die Vp. konnte am Ende des Versuches nicht mehr stehen. Oberste $\mathrm{Kurve}$ : Curaredosierung. Mittlere $\mathrm{K}$ urve: Temperatur im unteren Oesophagus. Un te r e K u r v e : Atemminutenvolumen, Mittelwerte über 2 min. (Nach KITZING \& BLEICHERT 1965)

Falle einer Irradiation efferenter Impulse von der motorischen Hirnrinde auf thermoregulatorische Zentren müßte dann bei gleicher Arbeit die Körpertemperatur stärker ansteigen als ohne Curare. Wir haben bei diesen Versuchen sehr kleine Arbeitsintensitäten von $3 \mathrm{mkp} / \mathrm{sec}$ gewählt, weil bei großen Arbeitsintensitäten die Versuchspersonen die Leistung während der Curareinfusion nicht durchbalten konnten. Bei sehr kleinen Leistungen, bei denen in Leerversuchen die Temperatur gerade etwas über das Aus- 
gangsniveau zu steigen beginnt, müßte sich im Falle einer Irradiation selbst ein minimaler Temperaturanstieg deutlich bemerkbar machen. Wir konnten aber in keinem Falle nach Curareinfusion eine über die zufälligen Schwankungen von $0,1^{0} \mathrm{C}$ hinausgehende Temperatursteigerung bemerken (Abb. 1 und 2).

Wenn wir auch - trotz eines negativen Ruheversuches - die Möglichkeit einer direkten Einwirkung des Curare auf die thermoregulatorischen Zentren oder Effek-
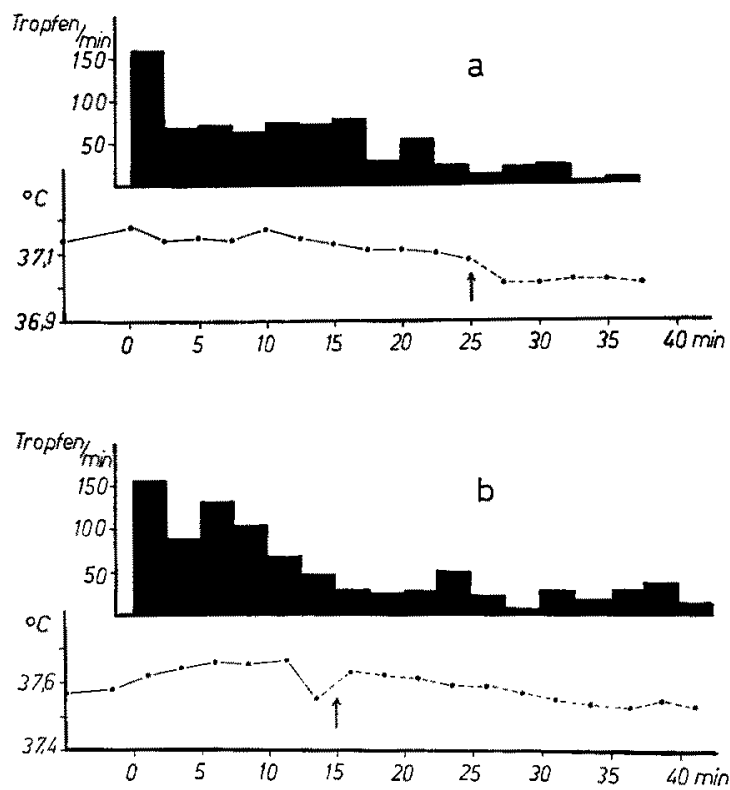

Abb. 2a und b: Zeitlicher Verlauf der Temperatur im unteren Oesophagus bei einer Leistung von $3 \mathrm{mkp} / \mathrm{sec}$ unter der Einwirkung von Curare. Beginn der Infusion im Zeitpunkt 0 . $\mathrm{Ob}$ e $\mathrm{K}$ urven: Mittelwerte der Curaredosierung. Untere $\mathrm{Ku}$ ryen: Temperatur im unteren Oesophagus. (a) Raumtemperatur $23^{\circ} \mathrm{C}$, relative Luftfeuchtigkeit $70 \%$. Mittelwerte aus vier Versuchen (ab Pfeil drei Versuche) an zwei Versuchspersonen. Mittelwert der Temperatur bei Arbeitsbeginn $37,2^{\circ} \mathrm{C}$. (b) Raumtemperatur $34^{\circ} \mathrm{C}$, relative Luffeuchtigkeit $50 \%$. Mittelwerte aus sechs Versuchen an drei Versuchspersonen (ab Pfeil drei Versuche an zwei Versuchspersonen). Mittelwert der Temperatur bei Arbeitsbeginn 37,40 C. (Nach Kitzing \& BLEICHERT 1965)

toren nicht ausschließen können, so halten wir es doch für unwahrscheinlich, daß ein etwaiger direkter oder indirekter Curareeffekt auf die Wärmeabgabe den möglichen Temperaturanstieg auf Grund einer Mitinnervation genau kompensiert.

Im folgenden soll untersucht werden, ob die Annahme einer Sollwertverstellung der Thermoregulation bei Arbeit überhaupt berechtigt ist. Im Falle einer Sollwertverstellung müßte auch in kalter Umgebung - außerhalb des Bereiches der physikalischen Regulation - die Kerntemperatur bei Arbeit zu den gleichen Werten ansteigen wie bei derselben Arbeit in normalen Klimabereichen. Um die in Kälte erhöhten Wärmeverluste auszugleichen, müßte also durch chemische Gegenregulation eine zusätzliche Stoffwechselsteigerung auftreten. Setzt diese chemische Gegenregulation erst 
dann ein, wenn die Körpertemperatur bei Arbeit unter die normale Temperatur bei Körperruhe zu sinken beginnt, so spricht dieser Punkt eindeutig gegen eine Sollwertverstellung. Tritt die Stoffwechselsteigerung aber erst auf, wenn die Temperatur unter den Arbeitswert zu sinken beginnt, so kann ein solcher Befund nur mit Vorsicht als ein Beweis für eine Sollwertverstellung ausgelegt werden, denn auch ohne eine gesenkte Blut- oder Gehirntemperatur kann die chemische Gegenregulation durch von der Haut ausgehende Reflexe ausgelöst werden. Die bisherigen Untersuchungen über die Abhängigkeit der Kerntemperatur bei Arbeit von den klimatischen Umgebungsbedingungen bewegen sich fast alle innerhalb des Bereiches der physikalischen Regulation.
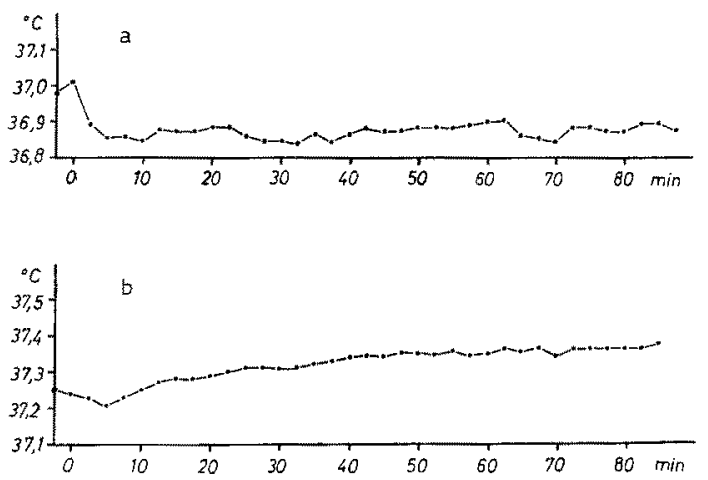

$A b b$. 3a und $b$ : Zeitlicher Verlauf der Temperatur im unteren Oesophagus bei einer Leistung von $3 \mathrm{mkp} / \mathrm{sec}$. Beginn der Arbeit im Zeitpunkt $O$. (a) Mittelwerte von fünf Versuchen an drei Versuchspersonen, Raumtemperatur $230 \mathrm{C}$, relative Luffeuchtigkeit $70 \%$. (b) Mittelwerte von acht Versuchen an drei Versuchspersonen, Raumtemperatur $34^{\circ} \mathrm{C}$, relative Luftfeuchtigkeit $50 \%$. (Nach KitZING \& BLEICHERT 1965)

NIESLEN (1938) schildert allerdings einen Versuch bei $8^{\circ} \mathrm{C}$ Umgebungstemperatur und sehr starker Luftbewegung, bei welchem bei einer Arbeit von $15 \mathrm{mkp} / \mathrm{sec}$ die Kerntemperatur um ein halbes Grad weniger angestiegen ist als bei normalen Klimabedingungen. Da der Bereich der physikalischen Regulation mit steigender Arbeit und damit steigender Stoffwechselleistung in Richtung zu kälteren Umgebungstemperaturen verschoben und gleichzeitig verbreitert wird, müßte umgekehrt bei sehr kleinen Arbeitsintensitäten schon in wesentlich wärmerer Umgebung eine möglicherweise verminderte Körpertemperatur zu beobachten sein. Wir konnten bei unseren Kontrollversuchen $\mathrm{zu}$ den Curareexperimenten bei $23^{\circ} \mathrm{C}$ Außentemperatur sogar ein Abfallen der Kerntemperatur während Arbeit $(3 \mathrm{mkp} / \mathrm{sec}$ ) unter den Ruhewert feststellen. Nur bei einer übergewichtigen Versuchsperson $(175 \mathrm{~cm}, 85 \mathrm{~kg})$ trat unter diesen Versuchsbedingungen eine leichte Arbeitshyperthermie auf. Bei $34^{\circ} \mathrm{C}$ Umgebungstemperatur steigt dagegen die Kerntemperatur bei derselben Arbeit stets geringfügig an (Abb. 3). Der Temperaturabfall unter das Ruheniveau bei unseren Versuchen bei $23^{\circ} \mathrm{C}$ erklärt sich wohl durch die gegenüber Ruhe bei Arbeit verbesserten Wärmeübergangsund Transportbedingungen durch die vermehrte Körperbewegung der Versuchsperson.

Wir halten nach unseren bisherigen Versuchsergebnissen eine Sollwertverstellung bei Muskelarbeit zumindest für sehr fraglich. Ebenso wie man eine Sollwertver- 
stellung annimmt, kann man die Arbeitshyperthermie als bleibende Abweichung bei einem Proportionalregler auffassen. Für diese Annahme muß dann nur gefordert werden, daß die Bluttemperatur als Reiz für die Auslösung von Wärmeabgabemechanismen wirken kann. Daß dies tatsächlich der Fall ist, dürfte wohl nach den bisherigen Ergebnissen als sicher gelten. Zusätzlich können natürlich auch noch andere Faktoren als Reiz für die Zentren in Betracht kommen. $\mathrm{Ob}$ außer der Bluttemperatur, zentralnervöse Einflüsse oder periphere Wirkungen von den Thermorezeptoren der Haut oder von tiefen im Körperkern gelegenen Thermorezeptoren die Empfindlichkeit der Zentren der Wärmeabgabe auf einen Reiz verändern können, muß nach den bisherigen Ergebnissen als offen gelten. So wäre zum Beispiel - entsprechend der von BRENDEL (1960) nachgewiesenen Empfindlichkeitsverminderung in Hypothermie - eine Empfindlichkeitszunahme durch eine erhöhte Bluttemperatur denkbar. Andererseits können die zentralnervösen Strukturen schon bei normalen Temperaturen ihre maximale Empfindlichkeit erreicht haben. Daß die Kerntemperatur in einem gewissen Bereich bei Arbeit ebenso wie in Ruhe fast unabhängig von den klimatischen Umgebungsbedingungen ist, zeigt nur, daß zusätzlich zu einer zentralen Anregung der Wärmeabgabe entsprechend der gesteigerten Wärmebildung die physikalische Regulation über die Haut genauso wie in Ruhe vor sich geht. Die Klimaunabhängigkeit der Arbeitshyperthermie erklärt sich damit durch die Störgrößenaufschaltung an den Thermorezeptoren der Haut.

Nachtrag: Inzwischen haben NIELSEN \& Nrelsen (1965) auch bei passiver Hyperthermie (Aufheizung ruhender Versuchspersonen mit Diathermie) eine Klimaunabhängigkeit wie bei der Arbeitshyperthermie gefunden. Auch dieser Befund läßt sich - ohne die Annahme einer Sollwertverstellung - als bleibende Abweichung in einem P-Reglersystem mit Störgrößenaufschaltung deuten.

\section{ZUSAMMENFASSUNG}

1. Die Klimaunabhängigkeit der Arbeitshyperthermie führte zu der Annahme einer Sollwertverstellung thermoregulatorischer Schaltstellen durch irradiierende motorische Impulse oder proprioceptive Rückmeldungen.

2. Folgende Befunde sprechen gegen diese Annahmen: (a) Unter der Einwirkung von Curare ändert sich die Arbeitshyperthermie trotz vermehrter efferenter Impulse nicht. (b) Bei kleiner Leistung ( $3 \mathrm{mkp} / \mathrm{sec}$ ) ist schon bei $23^{\circ} \mathrm{C}$ Außentemperatur ein Abfall der Kerntemperatur nachweisbar.

3. Es wird versucht, die Arbeitshyperthermie als bleibende Abweichung in einem P-Regelsystem, die Klimaunabhängigkeit als Folge der Störgrößenaufschaltung an den Thermoreceptoren der Haut zu erklären.

\section{ZITIERTE LITERATUR}

Asmussen, E. \& Nielsen, M., 1947. Regulation of body temperature during work performed with the legs and with the arms. Acta physiol. scand. 14, 373. 
BRENDEL, W., 1960. Die Bedeutung der Hirntemperatur für die Kältegegenregulation. Pflügers Arch. ges, Physiol. 270, 607.

Kitzing, J. \& Bleichert, A., 1965. Untersuchungen zur Arbeitshyperthermie des Menschen. Pflïgers Arch, ges. Pbysiol, 282, 242.

Nielsen, B. \& Niersen M., 1965. Influence of passive and active heating on the temperature regulation of man. Acta physiol. scand. 64, 323.

Nrelsen, M., 1938. Die Regulation der Körpertemperatur bei Muskelarbeit. Skand. Arch. Pbysiol. 79, 193.

OChwadT, B., Bücheri, E., Kreuzer, H. \& LoeschcKe, H. H., 1959. Beeinflussung der Atemsteigerung bei Muskelarbeit durch partiellen neuromuskulären Block (d-Tubocurarinchlorid). Pflügers Arch. ges. Pbysiol. 269, 613.

RoBinson, S., 1949. In: Physiology of heat regulation and the science of clothing. Ed. by L. H. Newburgh. Saunders, Philadelphia, Pa, 193.

\section{Diskussion im Anschluß an den Vortrag Krtzing \& BLeICHerT}

BRÜCK: Ist Ihre Interpretation zwingend? Wie ist es möglich, daß ein curarisierter Mensch Arbeit leistet? Es wäre denkbar, daß infolge der Curarisierung die Wärmeproduktion in der Muskulatur nicht genügend hoch eingestellt werden kann, um den lstwert der Körpertemperatur dem nach dem Konzept von Nisson unter Arbeit erhöhten Sollwert nachzuführen.

Kitzing: Wir haben den Versuchspersonen soviel Curare injiziert, daß sie gerade noch arbeiten können $-z$ wischen 10 bis $15 \mathrm{mg}$ pro Versuchsperson. Diese verspürten, daß die Arbeit wesentlich schwerer war. Zumindest in den Versuchen bei $34^{\circ} \mathrm{C}$ Raumtemperatur müßte, auch ohne zusätzliche Umsatzsteigerung in der Muskulatur, durch Einschränkung der Wärmeabgabe und durch "non-shivering thermogenesis" ein Temperaturanstieg möglich sein.

ZEISBERGER: Did you also measure oxygen consumption?

KITZING: Der $\mathrm{O}_{2}$-Verbrauch liegt in den Curareversuchen schon vor Beginn der Infusion höher als bei den Kontrollversuchen (Aufregung der Versuchsperson). Nach Infusionsbeginn steigt der $\mathrm{O}_{2}$-Verbrauch weiter an. Als Ursache könnten die Fibrillationen der Muskulatur in Frage kommen.

DONHOFfer: Könnte bei Thren Versuchen nicht die geringe Intensität der geleisteten Arbeit eine Rolle spielen?

KITznNG: Gerade bei geringen Leistungen wird die Differenz zwischen dem Innervationsaufwand mit und ohne Curareinfusion wegen der möglichen höheren Dosierung besonders deutlich. 